\title{
Appendiceal Carcinoma with Peritoneal Dissemination: Outcomes for the Best of the Best
}

\author{
Edward A. Levine, MD \\ Surgical Oncology Service, Wake Forest University, Winston-Salem, NC 27157, USA
}

Cancer of the appendix is a rare neoplasm; only a few thousand cases per year are reported in the US. The average busy general surgeon will encounter a case approximately once per decade. When peritoneal dissemination occurs, as it often does, it may lead to mucinous ascites, also known as pseudomyxoma peritonei. Such peritoneal dissemination results in a slowly progressive process, which ultimately leads to death as a result of bowel obstruction. Most appendiceal cancers are low-grade neoplasms that are typically relatively indolent. This low-grade/indolent behavior has led some to suggest a nonaggressive approach to therapy. Despite this, a number of centers have chosen to pursue aggressive cytoreductive surgery and intraperitoneal chemotherapy.

In the August issue of the Annals of Surgical Oncology, one of the leading proponents of aggressive cytoreductive surgery with intraperitoneal chemotherapy for disseminated peritoneal surface disease from appendiceal tumors presents his experience. ${ }^{1}$ This is a landmark report of the outcomes of 402 patients treated with cytoreductive surgery and intraperitoneal chemotherapy, representing more than a decade's focused work in this area. This represents an enormous volume of work in patients with stage IV disease. The 10-year survival is a remarkable $85 \%$ in patients who are able to undergo complete cytoreduction with intraperitoneal chemotherapy for

Received June 26, 2007; accepted June 27, 2007; published online: August 21, 2007.

Address correspondence and reprint requests to: Edward A. Levine, MD; E-mail: elevine@wfubmc.edu

Published by Springer Science+Business Media, LLC ๑ 2007 The Society of Surgical Oncology, Inc. disseminated (predominantly) low-grade appendiceal carcinoma. However, it is critical to bear in mind what this means for appendiceal cancer patients, and even more importantly, what it does not.

Patients with peritoneal carcinomatosis from appendiceal cancers who are taken to the operating room for cytoreductive surgery must expect a formidable procedure. ${ }^{2-4}$ After aggressive efforts to remove all apparent disease, a complete (CCR0-1 or $\mathrm{R} 0-1)$ resection is achieved in approximately half of the cases. ${ }^{2,3}$ Those that can be completely cytoreduced represent the most favorable subset. Patients who cannot be adequately cytoreduced (debulked) fare much more poorly. ${ }^{2,4,5}$ Furthermore, this cohort consisted predominantly of tumors of low histological grade, which also has significantly better outcomes than high-grade appendiceal tumors. ${ }^{6}$ Thus, this cohort represents the best of the best of cases treated as part of an extensive experience of over 1000 patients with peritoneal surface disease at the Washington Hospital Center.

Other issues should be considered in the evaluation of this article. The long-term follow-up in this study is excellent. However, predicting 10 -year survival with mean follow-up of only 66 months may suggest somewhat better results than will actually be found with longer follow-up. Additionally, although patients received intraperitoneal hyperthermic chemotherapy (HIPEC or IPHC), additional early postoperative intraperitoneal chemotherapy (EPIC) was utilized selectively by criteria that are not crisply defined.

Despite these weaknesses, there are a number of important pieces of information to be mined from 
this article. First and foremost is that complete resection and intraperitoneal chemotherapy can yield excellent outcomes with prolonged disease-free survival. Clearly, the majority of the benefit arises from cytoreductive surgery. However, this begs the question of the value of the intraperitoneal chemotherapy; whether in the form of intraoperative intraperitoneal hyperthermic chemotherapy (HIPEC) or early postoperative intraperitoneal chemotherapy (EPIC). To date, a definitive randomized trial comparing cytoreductive surgery with or without IPHC (HIPEC) and/or EPIC has not been completed. While the disease-free survival in this study is superior to reports of cytoreductive surgery alone, this supports, but does not prove, its value. ${ }^{7}$

The $28 \%$ of cases that recurred after aggressive cytoreductive surgery in IP chemotherapy were typically treated with a repeat cytoreductive procedure and additional intraperitoneal chemotherapy. Repeat (second or even third) cytoreductive surgery are challenging procedures associated with similar outcomes to those of the initial procedure only if complete cytoreduction can be achieved. The use of cisplatinum and doxorubicin in recurrences in this setting is a curious choice as neither of these agents is particularly active against colorectal carcinoma. Despite this, the results support the use of repeat procedures when the disease does recur, if there is no extra-abdominal disease and complete cytoreduction is feasible. ${ }^{8}$ It is also noteworthy that the mean survival of those not chosen for repeat procedures is 30 months. This length of survival for unresected recurrent disease should be borne in mind when evaluating patients with recurrences.

The outcomes after cytoreductive surgery with IP chemotherapy are closely related to the completeness of cytoreduction. The high rates of complete cytoreduction with lower volumes of peritoneal disease [as measured by the peritoneal carcinomatosis index (PCI)] suggest that early intervention is appropriate. Patients presenting with voluminous mucinous ascites (pseudomyxoma peritonei) are much less likely to be able to undergo complete cytoreduction. Thus, when low-volume peritoneal disease is encountered, it does not seem prudent to wait for massive, symptomatic disease to develop before undertaking cytoreductive procedures. It should also be remembered that the mucinous ascites is a byproduct of the tumor, not the primary target of the cytoreduction per se; thus removal of the tumor producing the mucin is the primary goal of cytoreduction, not evacuation of accumulated mucinous ascites.
The use of systemic therapy is not discussed in this article. However, the 10-year overall survival achieved with cytoreductive surgery and intraperitoneal chemotherapy strongly refute a role of systemic chemotherapy in this setting. The observation that most of the peritoneal failures are potential candidates for further cytoreductive surgery with intraperitoneal chemotherapy also argues against a role for external beam radiation therapy. However, systemic therapy may be of value in patients with recurrent disease no longer amenable to cytoreduction.

Much has been learned and many centers have initiated programs for cytoreductive surgery and intraperitoneal therapy in the 13 years since the database in this article was initiated. This therapy requires aggressive surgery associated with a significant learning curve. ${ }^{9}$ Concentrating these cases at regional centers with experience of cytoreductive surgery, peritoniectomy procedures and intraperitoneal therapy should benefit patients. The rarity of appendiceal carcinoma makes randomized trials extremely difficult to complete. Nevertheless, those of us who regularly treat this disease need to martial our resources to initiate such trials.

\section{REFERENCES}

1. Yan TD, Bijelic L, Sugarbaker PH. Critical analysis of treatment failure following complete cytoreductive surgery and perioperative intraperitoneal chemotherapy for peritoneal dissemination from appendiceal neoplasms. Ann Surg Oncol 2007; 14:2289-2299; doi:10.1245/s10434-007-9462-0.

2. Stewart JH, Shen P, Russell GB, et al. Appendiceal neoplasms with peritoneal dissemination; outcome after cytoreductive surgery in intraperitoneal hyperthermic chemotherapy. Ann Surg Oncol 2006; 13:1-12.

3. Sugarbaker PH, Alderman R, Edwards G, Marquardt CE, Gushchin V, Esquivel J, Chang D. Prospective morbidity and mortality assessment of cytoreductive surgery plus perioperative intraperitoneal chemotherapy to treat peritoneal dissemination of appendiceal mucinous malignancy. Ann Surg Oncol 2006; 13:635-644.

4. Glehen O, Mohamed F, Sugarbaker PH. Incomplete cytoreduction in 174 patients with peritoneal carcinomatosis from appendiceal malignancy. Ann Surg 2004; 240:278-285.

5. Ronnett BM, Zahn CM, Kurman RJ, Kass ME, Sugarbaker $\mathrm{PH}$, Shmookler BM. Disseminated peritoneal adenomucinosis and peritoneal mucinosis carcinomatosis. A clinical pathologic analysis of 109 cases with emphasis on distinguishing pathologic features, site of origin, prognosis, in relationship to "Pseudomyxoma Peritonei". Am J Surg Pathol 1995; 19:1390-1408.

6. Bradley RF, Stewart JH, Russell G, Levine EA, Geisinger KR. Pseudomyxoma peritonei of appendiceal origin: a clinicopathologic analysis of 101 uniformly treated patients at a single institution, with literature review. Am J Surg Pathol 2006; 30:551-559.

7. Miner TJ, Shia J, Jaquest DP, et al. Long-term survival following treated of pseudomyxoma peritonei; An analysis of surgical therapy. Ann Surg 2005; 241:300-308. 
8. Levine EA. Problems of Success and Problems of Failure; Recurrent disease after cytoreductive surgery and intraperitoneal chemo perfusion. Ann Surg Oncol 2004; 11:351353.
9. Levine EA, Stewart JH, Russell GB, et al. Cytoreductive surgery and intraperitoneal chemotherapy for peritoneal surface malignancy; experience with 501 procedures. J Am Coll Surg 2007; 204:943-955. 